\title{
Transient FDTD Simulation Validation
}

\author{
Ricardo Jauregui, Pere J. Riu and Ferran Silva \\ Departament d'Enginyeria Electrònica (DEE), Grup de Compatibilitat Electromagnètica (GCEM), \\ Universitat Politècnica de Catalunya (UPC), Barcelona, Spain. \\ ricardojt@eel.upc.edu \\ priu@eel.upc.edu \\ ferran.silva@upc.edu
}

\begin{abstract}
In computational electromagnetic simulations, most validation methods have been developed until now to be used in the frequency domain. However, the EMC analysis of the systems in the frequency domain many times is not enough to evaluate the immunity of current communication devices. Based on several studies, in this paper we propose an alternative method of validation of the transients in time domain allowing a rapid and objective quantification of the simulations results.
\end{abstract}

\section{INTRODUCTION}

In recent years, computational electromagnetism has had a great development thanks to the computational systems speed increase and their cost reduction. With those improvements the mathematical algorithms are able to work properly with more practical EMC issues. The problem that arises many times is to become confident with the results, in other words, to be able to quantitatively validate the results of the numerical simulation.

Today we can find some available validation methods [1]. The aim of all methods is to quantify the similarity of two datasets, making them an objective tool for test engineers to discuss data on a similar base. They also offer the possibility of a quantitative assessment of the ranked results to be interpreted as an expert opinion [2] [3].

The validation method most widely used today because of its versatility and simplicity in the field of EMC, is the Feature Select Validation method (FSV) [4] [5]. FSV has the advantage of analysing two major aspects that are considered in any validation, the different magnitude levels and of the graph shape.

Until now, most validation methods have been developed for comparison in the frequency domain. However, analysis of electronic systems only in the frequency domain is not enough to evaluate their immunity. One good example of the importance of the analysis in the time domain are the digital communications systems. These systems are very immune to continuous interference thanks to the modern techniques of channel coding and the complex protocols utilized, but the digital system becomes weak in the face of a radiated transient as shown by numerous studies [6][7][8].

Although FSV has a lot of features and a special module for the time domain, the method is not able to make a correct interpretation of the results when a transient pulse is analysed [9].

After developing an analysis about FSV performance in transient scenarios, this paper presents a new method to validate the numerical simulation of radiated transients in time domain. Five indicators are proposed to compare the two data sets. In this work we compare FDTD numerical simulations with actual transient interference measurements.

Finally, through an example, the validation method developed is compared with the FSV method to verify the quality of the indicators when transients signals are involved.

\section{FSV PERFORMANCE WITH TRANSIENTS}

FSV method is based on the decomposition of the results into two groups; the first one discusses the difference in amplitude (Amplitude Difference Measure, ADM) and the second one the difference between the characteristic signals (Feature Difference Measure, FDM). The combination of these two indicators (ADM and FDM) is a measurement of the overall difference (Global Difference Measure, GDM) [4] [5].

All indicators ADM, FDM and GDM have the ability to be configured to perform a point-to-point analysis. The advantage of relying on a point-to-point data is to know which areas of the data sets have the major differences. A subscript " $i$ " is added to consider this point-by-point feature (ADMi, FDMi and GDMi).

Another way to qualitative analyse the FSV indicators is represented by a probability density function. This indicator is useful for a rapid and comprehensive analysis of the results. Histograms are sorted according to the quality in excellent, very good, good, fair, poor and very poor.

Finally, a technique that has proved useful in presenting and interpreting FSV data, particularly the confidence histograms, is a "Grade and Spread" (G/S) diagram. The Spread serves a similar purpose to variance or standard deviation in statistical methods and is a measure of the spread of a distribution. The Grade is a measure of quality of the results and serves a similar purpose to skew measurements in statistics. It is important to remember that Grade and Spread must be used together, since if only one is used, the interpretation can be inaccurate [10]. 
This efficient and rapid method of analysis is ideal for most signals, but in the specific case of the transient signals, it has several drawbacks.

The main problem in using the FSV method for analyse a transient in the time domain arises from the magnitude indicator (ADM), which leads to an error in the global indicator (GDM) causing a misinterpretation of the results.

Usually, the transient pulses have a high peak of magnitude that decreases very rapidly in time. This particular settling time is the main cause the ADM indicator is not able to perform a correct interpretation.

To help to understand these limitations of the ADM Indicator when FSV method is applied [11], we have designed two test cases by comparing two transient signals that are defined by:

$$
\begin{aligned}
\text { Dat_set } & =e^{-\Delta t}\left[\left(2 \times 10^{-4}\right) * \operatorname{Cos}\left(2 \pi 3 \times 10^{8}\right)+\left(2 \times 10^{-4}\right) * \operatorname{Sin}\left(2 \pi 3 \times 10^{8}\right)+\ldots\right. \\
+ & \left(2.2 \times 10^{-2}\right) * \operatorname{Cos}\left(2 \pi 1 \times 10^{8}\right)+\left(2.2 \times 10^{-2}\right) * \operatorname{Sin}\left(2 \pi 1 \times 10^{8}\right)+\ldots \\
& +\left(5 \times 10^{-4}\right) \operatorname{Cos}\left(2 \pi 5 \times 10^{7}+\left(5 \times 10^{-4}\right) * \operatorname{Sin}\left(2 \pi 5 \times 10^{7}\right)\right]
\end{aligned}
$$

Where $\Delta \mathrm{x}$ are changed to obtain the two different signals data sets: Dat_Set1 with $\Delta 1=80 \times 10^{6}$ and Dat_Set2 with $\Delta 2=40 \times 10^{6}$. The signals obtained are presented in Fig 1 .

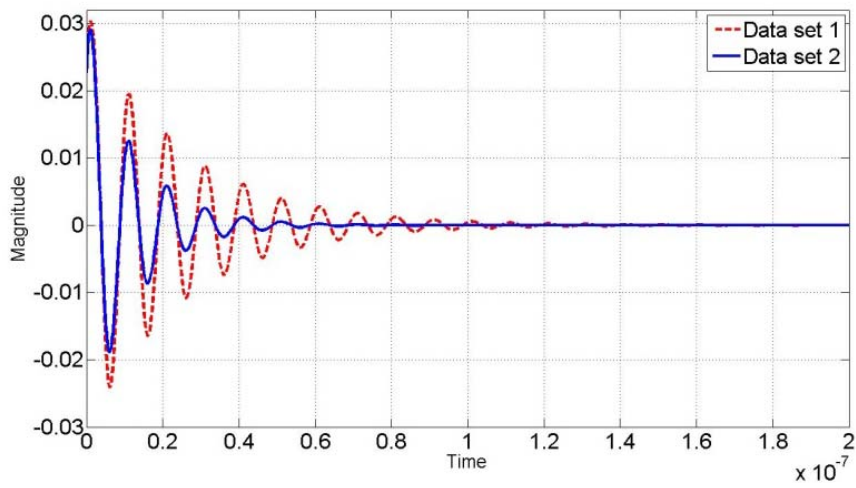

(a)

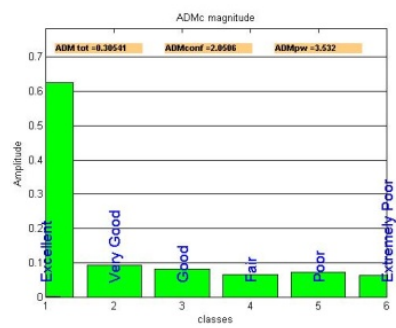

(b)

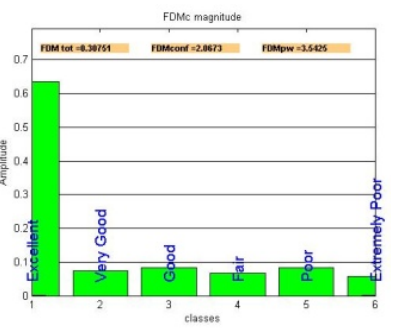

(c)
Fig 1. (a) First example result. (b) ADMc indicator. (c) FDMc indicator.

We could observe that, although there is a clear amplitude difference between both data sets, the FSV indicators Fig $1 \mathrm{~b}$ give us an "'Excellent" as a result, which does not correspond to the visual interpretation. The Grade and Spread for both the
$\mathrm{ADMc}$ and FDMc is equal to 4, showing a generally fair agreement and suggests the need for possible improvement of the results. Although this new indicator is closer to the visual perception, it is not easy to understand the real quality of the result.

In the second case $\Delta 1$ is changed to $10 \times 10^{6}$ which produces very different signal decay and $\Delta 2$ is maintained $\left(40 \times 10^{6}\right)$, as can be seen in Fig 2.

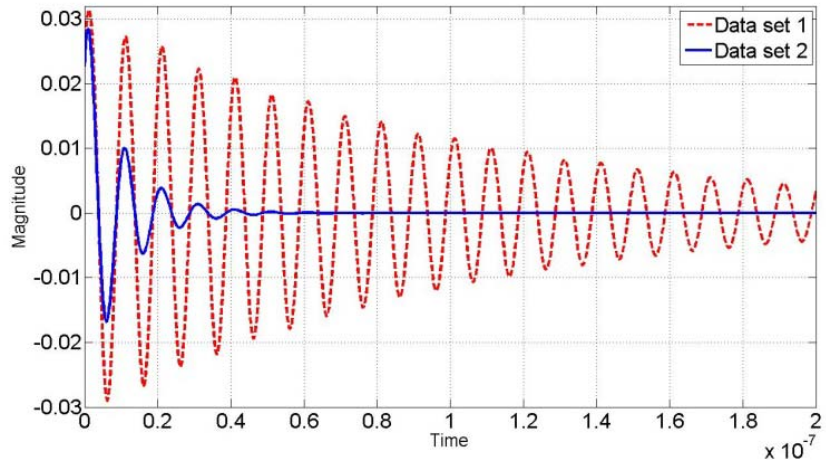

(a)

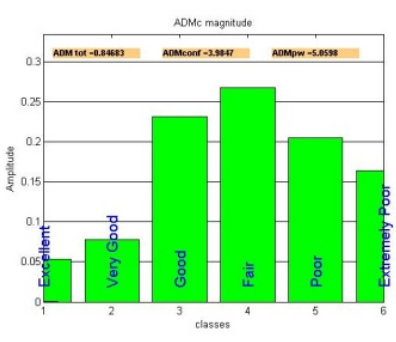

(b)

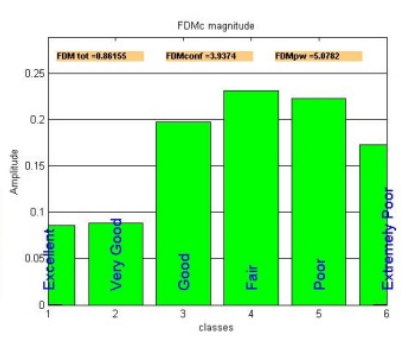

(c)
Fig 2. (a) Second example result. (b) ADMc indicator. (c) FDMc indicator.

In this second test case, the fault of the ADM indicator is even clearer. The difference in amplitude between both signals is evident; however the result of the ADM indicator is just "Fair".

Applying the "Grade and Spread" indicators for ADM and FDM we obtain $6 / 4$ and $6 / 5$, respectively. Again, the values are closer to the visual opinion, showing that it is very important use these indicators to have a completed results interpretation. However, despite all its advantages, it is difficult to know exactly what happens or where the transient has problems.

From the previous test cases analysed, we can realise that the $\mathrm{ADM}$ is the worst indicator, becoming the less reliable when the settling time of the signal is larger.

The problem with the ADM indicator lies in the way it calculates the dataset "lo" [3]. This dataset is obtained through the breakpoint (IBP), which is calculated with the $40 \%$ of the signal, because it assumes that most of the signal energy contents is within this range. But, many times in a transient signal, up to the $90 \%$ of the energy can be contained in the 
first peaks. Therefore it is highly probable that only the first peak of the transient is considered and the other low level differences are not taken into account when comparing with FSV method.

\section{TRANSIENTS VALIDATION NEW METHOD DEFINITION}

Based on several studies [7], we propose a new method to perform the Transient Time Domain Validation (TTDV). This method allows rapid and objective quantification of the simulation results, but it is important to note that this to study validation method is valid only the transient in time domain.. It is proposed to use five indicators to assess the different parameters of the transient data sets:
A. Feature Difference Measure (FDM).
B. Maximum Amplitude Levels (APL).
C. Maximum Rate Time (MRT).
D. Energy Contained in the Signals (ECS).
E. Total Error Average (TEA).

The fifth indicator (which includes the last three indicators: amplitude, rate time, energy), allows us a quick and easy interpretation of the results.

Following, the indicators of this method are explained in detail.

\section{A. Feature Difference Measure (FSV-FDM)}

This indicator reflects the difference or similarity between the shapes of both signals (Feature Difference Measure, FDM) The calculation of this indicator is made using the equations of the FSV method [4] in time domain. Unlike the amplitude indicator (ADM), the FDM does not present any problem when it is used to analyse the transient in time domain. Thanks to the different derivatives performed, it only takes into account the changing intervals in the graphs without attention to the level differences.

This indicator is applied before taking into account any other indicators, because its value determines whether or not to continue the validation process. After analysing different transient cases, we have determined that the optimal limit for a correct interpretation of the FDM indicator is about 0.8 . This value ensures that the two data sets (numerical simulation and measurement, for example) have a similarity that is within the acceptable margin.

If the FDM value is higher than 0.8 , or qualitative FDM is equal to "Poor" in most of the entire plot, none of the other indicators need to be applied, rejecting any further comparison between simulation and measurement.

\section{B. Amplitude Pulse Level (APL)}

The second indicator is the difference between the maximum amplitude of the signals (APL). The maximum level of a transient is often as important as the energy contained therein because it can produce several types of electronic equipment malfunctions. Thus, the APL indicator aims to assess the maximum amplitude level difference between the two data sets.

APL calculates the difference of the maximum of each data set in absolute value to guarantee that the analysis is independent of the polarity. The equations applied are (2) or (3), where $\max$ (a) is the maximum magnitude for the measured data and max (b) is the corresponding one for the simulated data set.

$$
\begin{array}{ll}
A P L=\frac{|\max (a)|-|\max (b)|}{\max (a)} & |\max (a)| \geq|\max (b)| \\
A P L=\frac{|\max (b)|-|\max (a)|}{\max (b)} & |\max (a)|<|\max (b)|
\end{array}
$$

APL range results in between 0 and 1 . Where there is a perfect similarity between the two plots the result will be 0 , but as they increase, the result moves to 1 .

\section{Maximum Rise Time (MRT)}

One important issue in a transient interference is the rise time. As lower as it is, more contents of the disturbance are on the high frequency band, which is usually a problem in EMC.

The calculation of this indicator is very similar to the one used in APL, the only difference is that it calculates the first derivative (4) and then applies the equations (5) or (6).

$$
\begin{aligned}
& D_{i}^{j}=\left(\frac{y_{i}^{j}-y_{i-1}^{j}}{x_{i}^{j}-x_{i-1}^{j}}\right) \quad \begin{array}{l}
j=\{a, b\} \\
i=\{1,2,3 \ldots n\} \quad \text { (4) }
\end{array} \\
& M R T=\frac{\left|\max \left(D^{a}\right)\right|-\left|\max \left(D^{b}\right)\right|}{\max \left(D^{a}\right)} \quad \max \left(D^{a}\right) \geq \max \left(D^{b}\right) \\
& M R T=\frac{\left|\max \left(D^{b}\right)\right|-\left|\max \left(D^{b}\right)\right|}{\max \left(D^{b}\right)} \quad \max \left(D^{a}\right)<\max \left(D^{b}\right)
\end{aligned}
$$

Where " $i$ " is the number of the point (from 1 to $n$ ). " $\mathrm{j}$ " is the set of the graph that we want to analyze ("a" is the measurement and "b" the simulation data set). $D_{i}^{j}$ is the derivative for each point (1 to n) of the measurement plot ("a") and simulated plot ("b"). $\operatorname{Max}(\mathrm{Da})$ is the absolute maximum value of the derivative of the measured values and $\max (\mathrm{Db})$ of the simulated ones. Similar to the APL indicator, we apply equation (5) or (6) in order to ensure that MTR varies from 0 to 1 .

\section{Energy Contained in the Signals (ECS)}

The energy contained in a transient signal is of vital importance since in many cases this will determine the damage it can produce to a victim system. 
Applying the equations (7) or (8), we can determine the error between the simulation and measurement data sets for the same interval of time. We calculate the difference between the energy values of the measured signal (a) and simulated (b) ones.

$$
\begin{array}{ll}
E^{j}=\int_{0}^{t_{n}} \frac{(U(t))^{2}}{R} d t & U=\{a, b\} \\
E C S=\frac{\left|E^{a}\right|-\left|E^{b}\right|}{\left|E^{a}\right|} & \left|E^{a}\right| \geq\left|E^{b}\right| \\
E C S=\frac{\left|E^{b}\right|-\left|E^{a}\right|}{\left|E^{b}\right|} & \left|E^{a}\right|<\left|E^{b}\right|
\end{array}
$$

Where $E$ is the energy and " $U$ " is the voltage recorded, both sets of measurements and simulation are defined from $\mathrm{t}=$ 0 to $\mathrm{t}=\mathrm{tn}$, assuming a resistance $\mathrm{R}$ equal to $50 \Omega$.

\section{E. The Total Error Average (TEA)}

When considering large numbers of data sets that must be compared, it is helpful to have one unique indicator that gives a general criterion of how good or bad the validation result might be. The indicator TEA meets this objective quickly and easily.

The calculation of TEA is based on finding the squared error of the indicators APL, MRT and ECS as shown in equation (10). In this equation a weighting factor for each indicator can be defined by " $\alpha$ ", " $\beta$ ", " $\gamma$ " to highlight the importance of a particular indicator in a particular situation. (In this paper they are set equal to 1 ).

$$
T E A=\sqrt{\frac{\alpha(A P L)^{2}+\beta(M R T)^{2}+\gamma(E C S)^{2}}{\alpha+\beta+\gamma}}
$$

To facilitate the validation process, different intervals are created to provide a quick inspection. Indicators APL, MRT, ECS and TEA can be classified as:

1. Good: from 0 to 0.3

2. Regular: from 0.3 to 0.5 .

3. Bad: from 0.5 to 1 .

Applying the indicators of the TTDV method to the previous examples in Section II (Fig 1 and 2), we obtain the table I.

TABLE I

TTDV RESULTS OF THE MEASUREMENTS AND SIMULATIONS.

\begin{tabular}{|c|c|c|c|c|c|}
\hline & \multicolumn{5}{|c|}{ TTDV indicators } \\
\cline { 2 - 6 } & FDM & APL & MRT & ECS & TEA \\
\hline Example 1 & 0.307 & 0.041 & 0.125 & 0.466 & 0.279 \\
\hline & & $R E G U L A R$ & $G O O D$ & $R E G U L A R$ & $G O O D$ \\
\hline Example 2 & 0.861 & 0.034 & 0.106 & 0.736 & 0.429 \\
\hline & & $R E G U L A R$ & $G O O D$ & $B A D$ & $R E G U L A R$ \\
\hline
\end{tabular}

As showing in Table I, with the TTDV method it is possible to detect that there are important differences between the signals with the overall factor: TEA $=0.279, \mathrm{TEA}=0.429$.

Thanks to the various indicators of the method it is possible to find that the major difference between the signals for both examples is the energy. In particular, in the second example, we can observe a very high level in this indicator, $\mathrm{ECS}=0.736$, which indicates that the signals being compared are substantially different.

\section{IV.APPLICATION TO FDTD MESHING OPTIMIZATION}

A serious problem when a numeric method is used arises when the most appropriate mesh for each simulation in order to obtain good results in the shortest time must be defined.

In this Section, the developed validation method is used to help us to find the most appropriate meshing value in a Finite Difference Time Domain (FDTD) numerical simulation. The FSV method and proposed TTDV method the will be used to compare real measurements with the different meshing FDTD simulations.

For this experiment, a resonant cavity is selected. The cavity is made of aluminium with dimensions of $246 \times 246 \times 100$ $\mathrm{mm}$. inside the cavity there are two monopoles with dimensions of $1.25 \mathrm{~mm}$ in diameter and $200 \mathrm{~mm}$ long. They are located at each end of the cavity, as shown in Figure Fig 3.

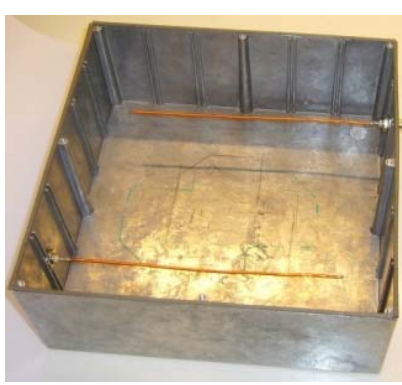

(a)

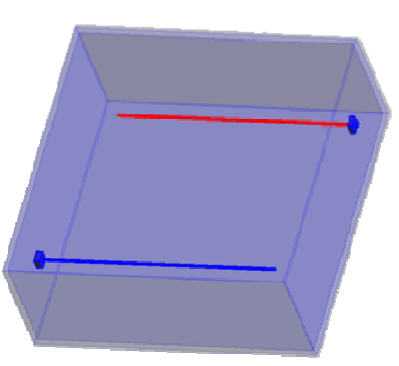

(b)
Fig 3. (a) Resonant Cavity without top. (b) FDTD Model.

\section{A. Measurements}

As we measure directly with an oscilloscope, we will apply a high level transient to avoid problems of sensitivity. A typical burst transient defined for EMC testing (EU-61000-4-4 [9]) and generated by Schölderl SFT 1400 is used. This signal has a bandwidth of approximately $300 \mathrm{MHz}$ and the selected magnitude is of $1 \mathrm{kV}$ as shown in Fig 4. 


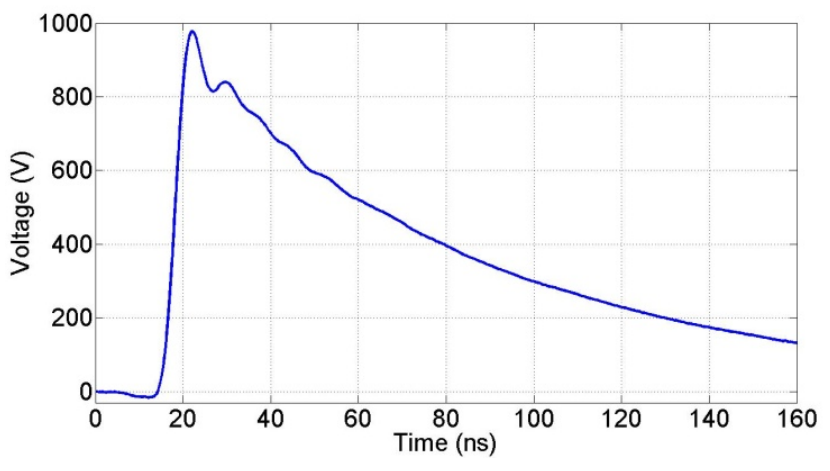

Fig 4. Transient pulse applied.

The oscilloscope chosen must have a bandwidth large enough to allow measuring the response of the smooth transition; in this case a Tektronix DPO7104 with a bandwidth of $1 \mathrm{GHz}$ with $50 \Omega$ and $500 \mathrm{MHz}$ with $1 \mathrm{M} \Omega$, with an ability to capture up to $20 \mathrm{GSA} / \mathrm{s}$ is used.

Special care has been taken in measuring the transient injected to the monopole and the coupled signal simultaneously. This is very important to obtain meaningful results because the injected transient will be used as input signal in the numerical simulation.

\section{B. FDTD Simulations}

The simulations were done using commercial software (SEMCA X [10]). This software uses the method of finite differences in the time domain (FDTD). The computer used was an Intel Corel Duo 2.66 GB 8 GB of RAMM.

Some post processing is needed before making any comparison between simulation and measurement. First of all it is necessary to apply a low pass filter with a cutoff frequency equal to the limit of the oscilloscope bandwidth to all the data sets, otherwise these out of band components could produce a wrong validation.

Finally, once the signal is filtered, it is necessary to apply a cross-correlation between simulation and measurement and to correct any small delay that may have occurred with the measurement instruments.

Three different meshing configurations were used. In the first one a $1 \mathrm{~mm}$ mesh for the critical zones of the model (monopole, source, load) and a $15 \mathrm{~mm}$ mesh for the other zones (air, cavity, etc) is used. This configuration gives a total of 680 cells. The results are obtained very quickly, but they have low quality (Fig 5).

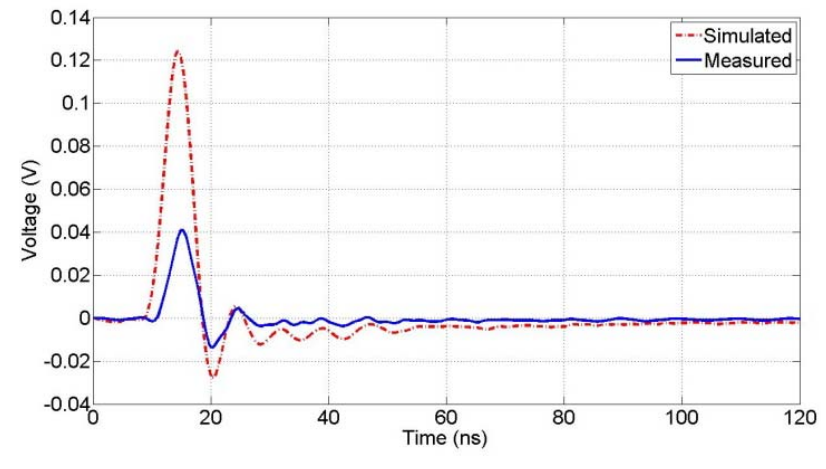

Fig 5. Comparison between measured and simulated (first configuration).

In the second configuration, the size of the cells was decreased to $0.2 \mathrm{~mm}$ in the critical zones of the model. With this new configuration a total of 0.36 MCell cells are obtained. In this case the results have been improved considerably (Fig $6)$.

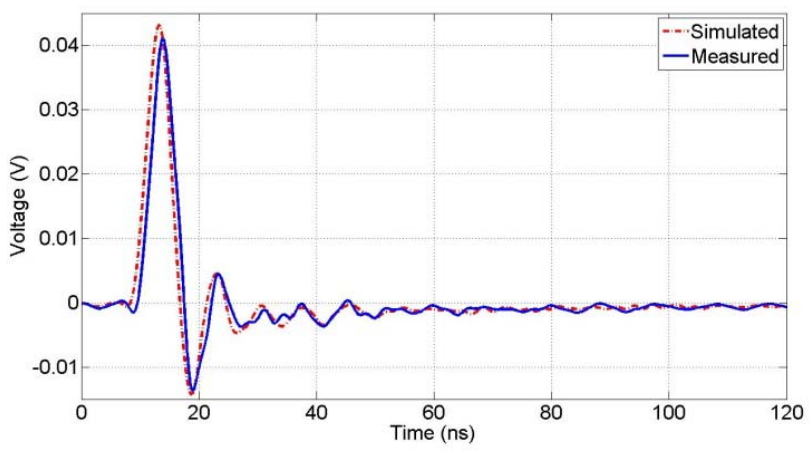

Fig 6. Comparison between measured and simulated (second configuration).

Finally, in the third configuration (Fig 7) a manual adjustment of the mesh was made in the monopole and at the edges of the cavity. In this case, the total number of cells increases to $0.61 \mathrm{MCell}$.

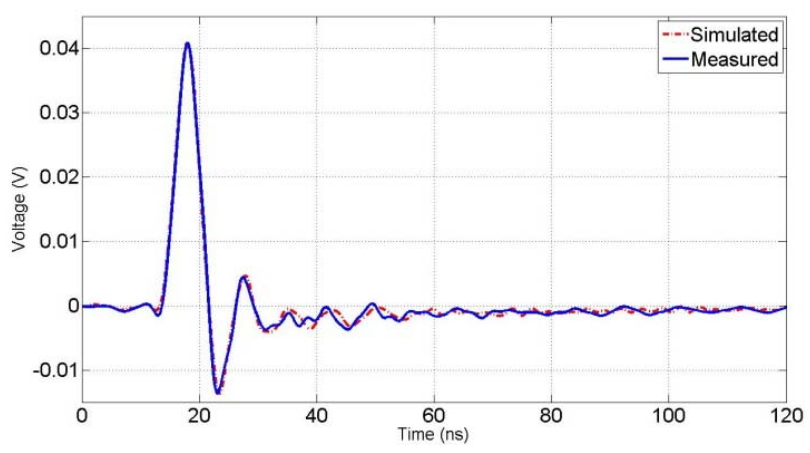

Fig 7. Comparison between measured and simulated (third configuration).

\section{Validation with FSV}

Table II show a total average of ADM, FDM and GDM indicators for the three different meshing configurations considered. As expected, the worst case is for the first configuration, which uses a very poor meshing. Looking at the 
global indicator we could see that it is classified as fair-poor $(\mathrm{GDM}=0.62)$. Undoubtedly this indicates that an improvement in the numerical model is necessary.

TABLE II

FSV RESULTS OF THE MEASUREMENTS AND SIMULATIONS.

\begin{tabular}{|l|c|c|c|c|c|}
\hline \multirow{2}{*}{} & \multicolumn{5}{|c|}{ FSV Indicators } \\
\cline { 2 - 6 } & ADM & FDM & GDM & G/S $_{\text {ADM }}$ & G/S $_{\text {FDM }}$ \\
\hline $\mathbf{6 8 0}$ Cell & 2.632 & 0.761 & 0.624 & $6 / 4$ & $5 / 5$ \\
\hline $\mathbf{0 . 3 6}$ MCell & 0.121 & 0.202 & 0.256 & $3 / 3$ & $3 / 3$ \\
\hline $\mathbf{0 . 6 1}$ MCell & 0.119 & 0.196 & 0.253 & $3 / 3$ & $3 / 3$ \\
\hline
\end{tabular}

The second case shows an important improvement compared to the previous one, declining the GDM indicator to 0.256 . Now the results of the simulation are classified for FSV as "Very Good".

The manual meshing improvements in critical areas did in the last configuration produces a small decrease in the indicator GDM of $1.1 \%$. Moreover, the G/S indicators are unable to indicate any difference between the improvements, so with this information one might conclude that it is not worthing an increase of 0.25 MCells for so little improvement.

\section{Validation with TTDV}

Table III shows the TTDV method results for the three meshing configurations. It can be seen how the TEA decreases when the quality of the mesh increases.

TABLE III

TTDV RESULTS OF THE MEASUREMENTS AND SIMULATIONS.

\begin{tabular}{|l|l|l|l|l|l|}
\hline & \multicolumn{5}{|c|}{ TTDV indicators } \\
\cline { 2 - 6 } & FDM & APL & MRT & ECS & TEA \\
\hline $\mathbf{6 8 0}$ Cell & 0.752 & 0.663 & 0.624 & 0.905 & 0.746 \\
\hline $\mathbf{0 , 3 6}$ MCell & 0.202 & 0.078 & 0.083 & 0.18 & 0.123 \\
\hline $\mathbf{0 , 6 1}$ MCell & 0.196 & 0.049 & 0.055 & 0.14 & 0.091 \\
\hline
\end{tabular}

In the first configuration, all indicators of the TTDV method (APL, MRT, ECS and TEA) are in the "bad" range. This result indicates that the simulation is not good enough and, like FSV results suggest, an improvement of the numerical model is needed. In the second and third configurations the results improve considerably respect to the first one, as expected.

The main difference when using the new TTDV proposed method is that we can identify a different behaviour between the two last configurations. Now the TEA value shows a decrease of $26 \%$ which allows us to quantify the difference between the results plotted in Figure Fig 6 and 7.

The proposed indicators can also provide further detailed analysis about the comparison carried out. In this case, the APL and MRT indicators show the largest changes from the second to the third configurations. This means that amplitude levels and rise time are the improved parameters achieved with the last meshing refinement.

\section{CONCLUSIONS}

A new easy-to-use validation method has been developed to compare data sets of transient phenomena like for example, numerical simulations and experimental measurements.

It has been shown that other methods like FSV fails in some transient cases when the behaviour of the signal is very different to the regular frequency plots.

In this paper we propose a simple mechanism for ranking correlations between pairs of results in time domain. It was also demonstrated that the value of a single indicator (TEA) is a very convenient quantitative tool when comparing many sets of data. Additionally, the definition of other meaningful indicators helps us to perform detailed EMC analysis of the transient signals.

\section{REFERENCES}

[1] A.L. Drozd, "Selected methods for validating computational electromagnetic modeling techniques," Electromagnetic Compatibility, 2005. EMC 2005. 2005 International Symposium on, 2005, pp. 301-306Vol.1.

[2] D.E. Coleby and A.P. Duffy, "Analysis of techniques to compare complex data sets," The current issue and full text archive of this journal is available, vol. 21, 2002, pp. 540-553.

[3] Duffy, A., Coleby, D., Martin, Woolfson, M., and T. Benson, "Progress in quantifying validation data," Electromagnetic Compatibility, 2003 IEEE International Symposium on, 2003, pp. 323-328vol.1.

[4] A.P. Duffy, A.J. Martin, A. Orlandi, G. Antonini, T.M. Benson, and M.S. Woolfson, "Feature selective validation (FSV) for validation of computational electromagnetics (CEM). part I-the FSV method," Electromagnetic Compatibility, IEEE Transactions on, vol. 48, 2006, pp. 449-459.

[5] A. Orlandi, A.P. Duffy, B. Archambeault, G. Antonini, D.E. Coleby, and S. Connor, "Feature selective validation (FSV) for validation of computational electromagnetics (CEM). part II- assessment of FSV performance," Electromagnetic Compatibility, IEEE Transactions on, vol. 48,2006 , pp. 460-467.

[6] P.J. Riu, R. Jauregui, S. Verdaguer, R. Santos, and F. Silva, "Transient Radiated Fields in 14/42V Powernet Systems FDTD assessment," EMC Europe 2006, Barcelona - Espaa, 2006.

[7] I.E. Portugues, P.J. Moore, I.A. Glover, and R.J. Watson, "A Portable Wideband Impulsive Noise Location System," vol. 57, 2008, pp. 20592066.

[8] J. Lago-Fernndez and J. Salter, R\&D White Paper Modelling impulsive interference in DVB-T: statistical analysis, test waveforms \& receiver performance.

[9] R.J. Telleria, F. Silva, A. Orlandi, H. Sasse, and A. Duffy, "Factors influencing the successful validation of transient phenomenon modelling," Asia-Pacific Electromagnetic Compatibility Symposium and Technical Exhibition. Beijing, 2010., 2010, pp. 2-5.

[10] B.A. Duffy, A. Orlandi, and A.P. Duffy, "Using the Feature Selective Validation Technique to Compare Data Sets," Electrical Engineering, 2009, pp. 248-253.

[11] University of L'Aquila EMC Laboratory (http://ing.univaq.it/uaqemc/FSV_4_0_3L/), "FSV 4.0." 